\title{
Actin-Capping Protein and the Hippo pathway regulate F- actin and tissue growth in Drosophila
}

\author{
Beatriz García Fernández, Pedro Gaspar, Catarina Brás-Pereira, Barbara Jezowska, Sofia Raquel Rebelo and \\ Florence Janody*
}

\begin{abstract}
SUMMARY
The conserved Hippo tumor suppressor pathway is a key kinase cascade that controls tissue growth by regulating the nuclear import and activity of the transcription co-activator Yorkie. Here, we report that the actin-Capping Protein $\alpha \beta$ heterodimer, which regulates actin polymerization, also functions to suppress inappropriate tissue growth by inhibiting Yorkie activity. Loss of Capping Protein activity results in abnormal accumulation of apical F-actin, reduced Hippo pathway activity and the ectopic expression of several Yorkie target genes that promote cell survival and proliferation. Reduction of two other actin-regulatory proteins, Cofilin and the cyclase-associated protein Capulet, cause abnormal F-actin accumulation, but only the loss of Capulet, like that of Capping Protein, induces ectopic Yorkie activity. Interestingly, F-actin also accumulates abnormally when Hippo pathway activity is reduced or abolished, independently of Yorkie activity, whereas overexpression of the Hippo pathway component expanded can partially reverse the abnormal accumulation of F-actin in cells depleted for Capping Protein. Taken together, these findings indicate a novel interplay between Hippo pathway activity and actin filament dynamics that is essential for normal growth control.
\end{abstract}

KEY WORDS: Capping Protein, Hippo signaling pathway, Actin cytoskeleton, Drosophila discs epithelia

\section{INTRODUCTION}

The conserved Hippo (Hpo) signal transduction pathway has emerged as a crucial regulator of tissue size in both Drosophila and mammals (Edgar, 2006). In Drosophila, the Hpo pathway comprises a kinase cascade in which the Hpo kinase binds the WW domain adaptor protein Salvador (Sav) to phosphorylate and activate the Warts (Wts) kinase (Pantalacci et al., 2003; Tapon et al., 2002; Udan et al., 2003; Wu et al., 2003). Wts, in turn, facilitated by Mats (Lai et al., 2005; Wei et al., 2007), phosphorylates and prevents nuclear translocation of the transcriptional co-activator Yorkie (Yki) (Huang et al., 2005; Oh and Irvine, 2008). This leads to transcriptional downregulation of target genes that positively regulate cell growth, survival and proliferation, including the Drosophila inhibitor of apoptosis protein 1 (Diap1; thread - FlyBase), expanded (ex), Merlin (Mer) and wingless $(\mathrm{wg})$ in the inner distal ring, within the proximal wing imaginal disc (Reddy and Irvine, 2008). The upstream components Ex, Hpo and Wts are also thought to regulate Yki through a phosphorylation-independent mechanism, by directly binding to Yki, sequestering it in the cytosol and thereby abrogating its nuclear activity (Badouel et al., 2009; Oh et al., 2009).

Multiple upstream inputs are known to regulate the core Hpo kinase cassette at various levels (Grusche et al., 2010; Halder and Johnson, 2010). Thus, the atypical cadherin Fat was identified as an upstream component of the Hpo pathway and was proposed to transduce signals from the atypical cadherin Dachsous (Ds) and Four-jointed (Fj), a Golgi-resident kinase that phosphorylates Fat

Instituto Gulbenkian de Ciência, Rua da Quinta Grande 6, P-2780-156 Oeiras, Portugal.

*Author for correspondence (fjanody@igc.gulbenkian.pt)

Accepted 9 March 2011 and Ds (Bennett and Harvey, 2006; Cho et al., 2006; Hamaratoglu et al., 2006; Silva et al., 2006; Willecke et al., 2006). Moreover, the two Ezrin-Radixin-Moesin (ERM) family members, Ex and Mer have been reported to lie upstream of the Hpo pathway (Hamaratoglu et al., 2006). Mer and Ex can heterodimerize (Badouel et al., 2009; Boedigheimer et al., 1993; Bretscher et al., 2002; McCartney et al., 2000) and are believed to exert their growth suppression activity by activating the Hpo kinase. However, how the different inputs that feed into the core kinase cassette are coordinated to regulate Yki activity is unknown.

ERM proteins form a structural linkage between transmembrane components and actin filaments (F-actin) (McClatchey and Giovannini, 2005). For instance, mammalian Mer binds numerous cytoskeletal factors, including actin, and appears to act as an inhibitor of actin polymerization (McClatchey and Giovannini, 2005; Scoles, 2008). Interestingly, the Merlin-actin cytoskeleton association is required for growth suppression and inhibition of epidermal growth factor (EGFR) signaling (Curto and McClatchey, 2008). Moreover, F-actin depolymerization promotes activation of the Hpo orthologs MST1 and MST2 in mouse fibroblasts (Densham et al., 2009). These observations suggest a role for Factin dynamics in modulating Hpo pathway activity.

Actin filament growth, stability and disassembly are controlled by a plethora of actin-binding proteins. Among these, the Capping Protein (CP) heterodimer, composed of $\alpha(\mathrm{Cpa})$ and $\beta(\mathrm{Cpb})$ subunits, acts as a functional heterodimer to restrict the accessibility of the filament barbed end, inhibiting addition or loss of actin monomers (Cooper and Sept, 2008). In Drosophila, mutations in either $c p a$ or $c p b$, lead to accumulation of F-actin within the cell and give rise to identical developmental phenotypes that are tissue specific. In the wing blade (BL), the most distal domain of the imaginal disc, $c p a$ and $c p b$ prevent cell extrusion and death, but they are not required for this function in the most proximal domain, the prospective body wall and the hinge wing imaginal disc (Janody and Treisman, 2006). The Cofilin homolog 
Twinstar (Tsr) and the Cyclase-associated protein Capulet (Capt) also restrict actin polymerization: Tsr severs filaments and enhances dissociation of actin monomers from the pointed end (Bamburg, 1999), whereas Capt sequesters actin monomers, preventing their incorporation into filaments (Gottwald et al., 1996).

Here, we investigate the relationship between the control of the actin cytoskeleton and Hpo pathway activity. We show that the actin-binding proteins $\mathrm{CP}$ and Capt, but not Tsr, enhance Hpo signaling activity. Moreover, we uncover a new relationship between the Hpo pathway and the machinery that regulates F-actin, and reveal that Hpo signaling activity, like CP, limits F-actin accumulation at apical sites independently of Yki. Finally, we propose that regulation of an apical F-actin network by Hpo signaling activity and CP sustains Hpo pathway activity, thereby limiting Yki nuclear import and the activation of proliferation and survival genes.

\section{MATERIALS AND METHODS}

\section{Fly strains and genetics}

The $c p a, t s r$ and $c p b$ alleles, in addition to the UAS-HA-cpa transgenic flies and the generation of clones have been previously described (Janody and Treisman, 2006). Other fly stocks used were ex ${ }^{697}$ (lacZ reporter) (Hamaratoglu et al., 2006); $s a v^{3}$ (Tapon et al., 2002); $\mathrm{Mer}^{4}$ (LaJeunesse et al., 1998); ats $^{e 235}$ (Lai et al., 2005); ppo $^{42-47}$, a gift from D. Pan (Wu et al., 2003); $e x^{e l}$ (Boedigheimer et al., 1993); wts ${ }^{\text {lats } X 1}$ (Xu et al., 1995); UAS- $h p o^{\text {M11.I }}$ (Pantalacci et al., 2003); UAS-yki ${ }^{S 168 A}$ (Dong et al., 2007); UAS-yki, UAS-Mer ${ }^{l-600}$ (LaJeunesse et al., 1998); UAS-yki-GFP (Oh and Irvine, 2008); UAS-ex (Udan et al., 2003); UAS-Fat (Sopko et al., 2009); Diap 1::lacZ (Hay et al., 1995); sd-Gal4 (Klein and Arias, 1998); $h$ h-Gal4, a gift from T. Tabata (University of Tokyo, Japan); $a p-G a 14 ; t u b-G a 180^{\text {ts }}$ (Bloomington Stock Center, IN, USA); UAS-hpo-IR (Pantalacci et al., 2003); UAS-yki-IR ${ }^{4005 R-2}$; UAS-capt-IR ${ }^{5061 R-2}$ and UAS-wts-IR ${ }^{12072 R-1}$ (National Institute of Genetics, NIG, Japan); UAS-cpa-IR ${ }^{7009}$ (Vienna Drosophila Research Center, VDRC, Austria).

\section{Immunohistochemistry}

Third instar larval imaginal discs were stained with antibodies as described (Lee and Treisman, 2001). Antibodies used were mouse anti-Arm [N2 7A1, 1:10; Developmental Studies Hybridoma Bank (DSHB)], rabbit antiHth (1:500; A. Salzberg, Technion-Israel Institute of Technology, Haifa, Israel), guinea pig anti-Hth (1:3000; R. S. Mann, Columbia University Medical Center, New York, NY, USA), mouse anti-Nub (1:10; S. M. Cohen, Institute of Molecular and Cell Biology, Singapore), rabbit anti-Ex (1:200; A. Laughon, University of Wisconsin, Madison, WI, USA), guinea pig anti-Mer (1:4000; R. Fehon, University of Chicago, IL, USA); mouse anti-Wg (4D4, 1:10; DSHB), rabbit anti-Yki (1:100) (Oh and Irvine, 2008), rabbit anti-Caspase 3 (1:500; BD Bioscience), pMLC (1:10; Cell Signaling), mouse anti- $\beta$-galactosidase $(1: 200$; Promega), mouse anti-HA (1:5000; Covance), mouse anti-BrdU (1:200; Roche). Rhodamineconjugated Phalloidin (Sigma) was used at a concentration of $0.3 \mu \mathrm{M}$. BrdU labeling was assayed as described (Johnston and Schubiger, 1996). Secondary antibodies were from Jackson ImmunoResearch, used at 1:200 or Molecular Probes, used at 1:500. Fluorescence images were obtained on a Leica TCS NT or a LSM 510 Zeiss confocal microscope. The NIH Image $\mathrm{J}$ program was used to perform measurements. To measure roundness, clones recovered in the proximal wing epithelium that were either FRT40A, wild type or FRT42D, wild type or FRT40A, $c p b^{M 143}$ or FRT42D, $c p a^{107 E}$, were outlined and the circularity was calculated as $4 \pi \mathrm{A} / \mathrm{L}^{2}$, where $\mathrm{A}$ is the clone area and $\mathrm{L}$ is the perimeter. To measure the surface area of $c p b$ mutant clones with no GFP and of sibling twin spots with two copies of GFP, clones recovered in the proximal wing disc epithelia were outlined separately. The surface area for each clone was calculated in pixels and converted to $\mu^{2}$ (where 1024 pixels corresponds to $387.5 \mu \mathrm{m}$ ). To measure the length of the Nub-Hth expression domain, the apical surface of the Nub-Hth positive domains were outlined on optical cross-sections (blue lines in Fig. 1E-G) and the length was measured on each disc for each genotype. To quantify the intensity of BrdU signal, the posterior and anterior compartment of $h h>\mathrm{GFP}$ and $h h>c p a$ $I R^{C 10}$ wing discs were outlined separately for each disc and the intensity levels were calculated as the sum of the gray values of all the pixels in the selection divided by the number of pixels for each compartment. Values were then normalized with those of $h h>G F P$ control discs. To quantify the intensity of Phalloidin signals, a region of interest (ROI) of $100 \times 100$ pixels ( $\sim 40$ cells) was selected. The sum of the gray values was measured for each ROI, applied twice to the posterior and to the anterior compartments (on each side of the dorsal/ventral and anterior/posterior boundaries) on standard confocal sections comprising the apical surface. The ratio of signal between the posterior and anterior compartments was calculated for each disc. Statistical significance was calculated using a twotailed $t$-test.

\section{Western blotting}

Proteins were extracted from wing imaginal discs expressing UAS-fat, UAS-cpa- $I R^{D 4}$ or UAS-wts-IR ${ }^{12072 R-1}$ under the control of $s d$-GAL4, at $25^{\circ} \mathrm{C}$, using a lysis buffer $(50 \mathrm{mM}$ Tris $\mathrm{pH} 7.4,150 \mathrm{mM} \mathrm{NaCl}, 1 \mathrm{mM}$ EDTA pH 7.4, 1\% NP-40) in the presence of protease inhibitors (Roche) and phosphatase inhibitors (Sigma). After adding the lysis buffer, samples were frozen in liquid nitrogen and subsequently spun at $13,000 \mathrm{~g}$ for 15 minutes at $4^{\circ} \mathrm{C}$. Protein amounts in the supernatant were quantified by the Bradford method to normalize quantities of extract used for loading between $s d>w 1118$ discs and $s d>f a t ; \quad s d>c p a-I R^{D 4} ; \quad s d>w t s-I R$ overexpressing wing discs. Total extracts were prepared according to NuPAGE Bis-Tris Mini Gel Protocol (Invitrogen) and run on a $10 \%$ NuPAGE Bis-Tris Gel (Invitrogen). Wet electrotransfers of gels to nitrocellulose membranes (Amersham Hybond-P, GE Healthcare) were performed at $30 \mathrm{~mA}$ overnight at $4{ }^{\circ} \mathrm{C}$. Blots were blocked for 2 hours at room temperature in PBST $(0.2 \%$ TritonX-100 in PBS) containing $5 \%$ lowfat milk and incubated overnight at $4{ }^{\circ} \mathrm{C}$ in PBST containing $5 \%$ low-fat milk supplemented with rabbit anti-pYki ${ }^{\mathrm{S} 168}$ (1:1000) or rat anti-Yki (Yki ${ }^{69}$ 1:1000; N. Tapon, Cancer Research UK, London, UK) or mouse anti- $\beta$ Tub (E7; 1/2000; DSHB). Blots were washed four times in PBST and incubated for 1 hour in PBST containing 5\% low-fat milk supplemented with ECL anti-rabbit IgG-conjugated HRP or anti-rat IgG-conjugated HRP ECL antimouse IgG-conjugated HRP (1:5000 dilution; GE Healthcare). After four washes in PBST, blots were developed using Amersham ECL Plus Western Blotting Detection System (GE Healthcare). The same membrane was first probed with anti-pYki ${ }^{168}$, followed by Yki and anti-tubulin antibodies after stripping.

\section{Molecular biology}

To make the UAS-cpa-IR construct, a 447 bp DNA fragment was amplified by PCR from genomic DNA of Oregon flies, using the oligonucleotides dscpa $5^{\prime}$ (ATTATCTAGAGGTACCAGCTCTGTTTTTGGAAAGGC containing $X b a \mathrm{I}$ and $K p n \mathrm{I}$ sites) and $d s c p a 3^{\prime}$ (ATTATCTAGATTATTGCGTCTTCAGTTCCT containing a $X b a \mathrm{I}$ site) and subcloned into the $X b a I$ site of pWIZ (Lee and Carthew, 2003) to give rise to the pWIZcpa3447 construct. The same PCR product digested with $X b a \mathrm{I}$ was then subcloned into the SpeI site of the pWIZcpa3-447 construct. Off-targets were analyzed using dsCheck (http://dscheck.rnai.jp/) (Naito et al., 2005). No off-targets from 429 mers were predicted. Three independent transgenic lines (UAS-cpa-IR ${ }^{C 10}$, UAS-cpa-IR ${ }^{B 4}$ and UAS-cpa-IR ${ }^{D 4}$ ) were generated by standard methods (Spradling and Rubin, 1982).

\section{RESULTS \\ Capping Protein suppresses proliferation and overgrowth of proximal wing tissue}

To understand the role of CP in epithelia, we analyzed in more detail groups of cells mutant for $c p a$ and $c p b$ in the proximal wing imaginal disc, where cell polarity and organization of the epithelium monolayer is not affected (Janody and Treisman, 2006). We noticed that the boundaries of mutant clones were abnormally straight (Fig. 1A), and the clones were also rounder than their wild- 

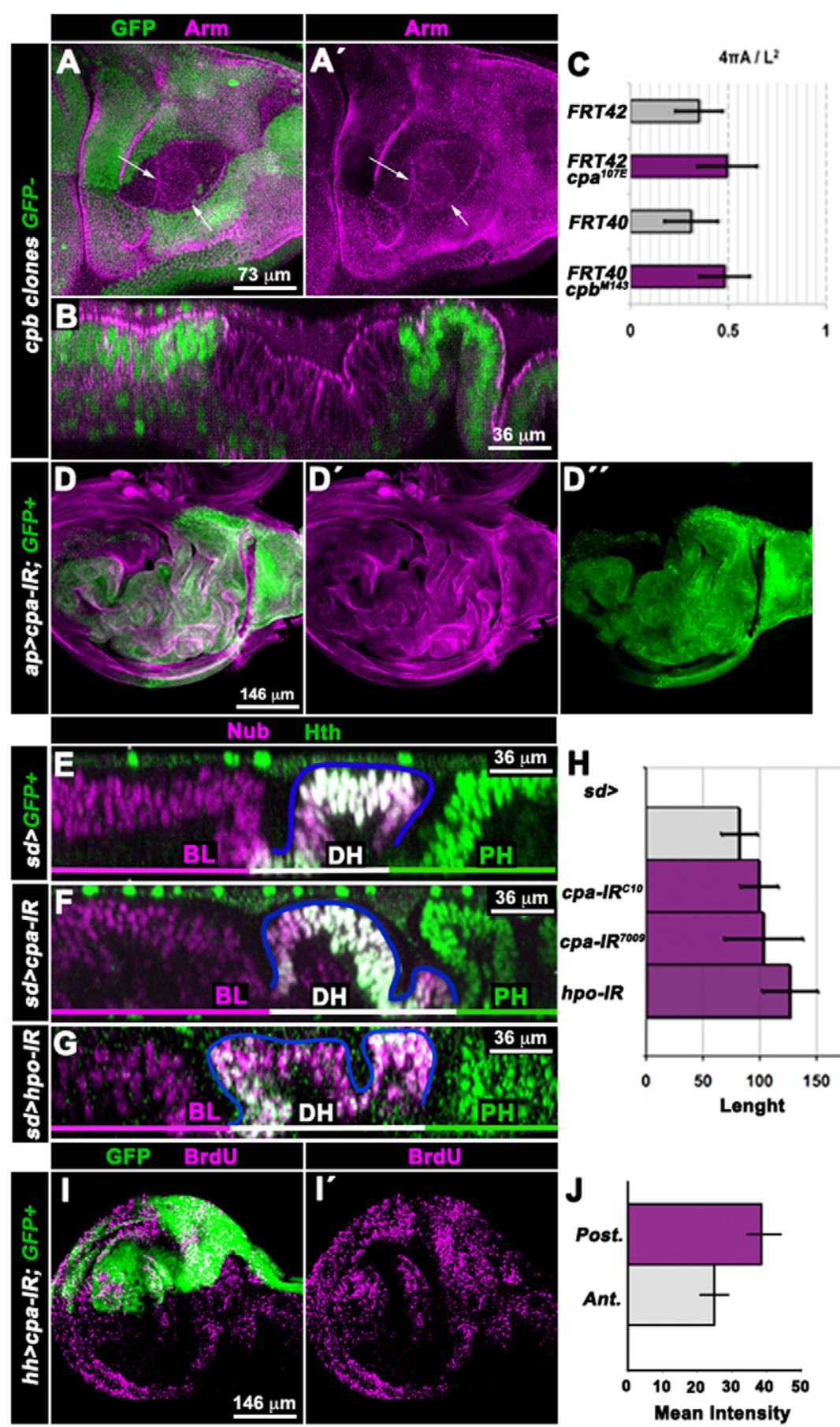

Fig. 1. Loss of CP promotes cell proliferation. (A$\left.B, D-G, I, I^{\prime}\right)$ Drosophila third instar wing imaginal discs shown as standard confocal sections (A, $\left.A^{\prime}, D-D^{\prime \prime}, I, I^{\prime}\right)$ or optical cross-sections (B,E-G) through disc epithelia. (A-B) $c p b^{M 143}$ mutant clones marked by the absence of GFP (green in A and B) and stained with anti-Arm (magenta) to outline the apical cell membrane. Arrows in $A$ and $A^{\prime}$ indicate folds around and within the clone. (C) Circularity of cpa and cpb mutant clones compared with FRT40A or FRT42D wild-type clones, calculated as $4 \pi \mathrm{I} A / L^{2}$, where $A$ is the clone area and $\mathrm{L}$ is the perimeter. The mean for FRT40A clones is 0.30 , for FRT40A cpb clones is 0.48 , for FRT42D clones is 0.34 and for FRT42D, cpa clones is 0.49 . Error bars indicate s.e.m. $P<0.001$ for comparison of FRT40A, wild type to FRT40A, $\mathrm{Cpb}^{\mathrm{M} 143}$ and FRT42D, wild type to FRT42D, cpa $107 \mathrm{E}$. (D-D") ap-Gal4 driving (D) UAS-GFP (green in D and $\left.D^{\prime \prime}\right)$ and UAS-cpa- $/ R^{C 10}$. Discs are stained with antiArm (magenta in $D$ and $D^{\prime}$ ). (E-G) sd-Gal4 driving UAS-GFP (E), UAS-cpa-IR ${ }^{C 10}$ (F) or UAS-hpo-IR (G). Discs are stained with anti-Nub (magenta) and anti$\mathrm{Hth}$ (green). The proximal domain, stained only with anti-Nub (magenta bar) represents the blade (BL). The domain stained with both anti-Nub and anti-Hth (white bar) represents the distal hinge (DH) and the domain stained only with anti-Hth (green bar) represents the proximal hinge $(\mathrm{PH})$. Solid blue lines outline the apical surface of the Nub/Hth-positive domains used to measure the length of the distal hinge domain in $\mathbf{H}$. (H) Measurement of the length of the apical surface of Nub/Hth-positive cells in $s d>c p a-I R^{C 10}$ or cpa-IR7009 or hpo-IR, compared with $s d>$ GFP control discs. The mean for $s d>$ GFP is 81.38 , for $s d>c p a-I R^{C 10}$ is 98.95 , for $s d>c p a-I R^{7009}$ is 102.8 and for $s d>h p o-I R$ is 126.13 . Error bars indicate s.e.m. $P<0.03$ for comparison of $s d>c p a-I R^{C 10}$ and $s d>$ cpa- $I R^{7009}$ to $s d>$ GFP and $P<0.001$ for comparison of $s d>h p o-I R$ to $s d>$ GFP. $\left(\mathbf{I}, I^{\prime}\right) h h-G a l 4$ driving UAS-GFP (green in I) and UAS-cpa-IR ${ }^{C 10}$. DiscS are labeled by BrdU incorporation (magenta).

(J) Mean intensity of BrdU signal in posterior versus anterior wing compartments of $h h>c$ cpa-IR ${ }^{C 10}$ after normalization with control $h h>$ GFP wing discs. The mean for posterior (Post.) cells is 24.96 and for anterior (Ant.) is 44.68. Error bars indicate s.e.m. $P<0.004$ for comparison of $A$ and $P$. type twin spots (Fig. 1C), suggesting that mutant cells minimize contacts with wild-type neighbors. When induced in first instar larvae, $40 \%$ of $c p b$ mutant clones displayed increased staining of the apical marker Armadillo (Arm) around or within the clones (arrows in Fig. 1A). Optical cross-sections through the wing disc showed that the mutant tissue was folded, but maintained the columnar shape of a polarized epithelium (Fig. 1B). Fold formation is therefore unlikely to be caused by a change in cell shape but might result from excess proliferation, giving rise to cyst-like clones that appear rounder than wild-type clones. To evaluate the growth rate of cells mutant for $c p b$, we induced mutant clones at first instar larvae and measured in the proximal wing domain, the surface area of $c p b$ mutant clones with no GFP, compared with their wild-type twin spots that contain two doses of GFP. $c p b$ mutant clones were on average $25 \%$ larger than wild-type twin spots (see Fig. S1A in the supplementary material). However, a mixture of very small (blue arrow in Fig. S1B in the supplementary material) and big clones (blue asterisk in Fig. S1B in the supplementary material) were recovered, suggesting that only some $c p b$ mutant clones could overgrow.

To further assess the cellular requirement for $\mathrm{CP}$, we generated stable lines of transgenic flies carrying an inverted repeat construct capable of expressing intron-spliced hairpin dsRNA for cpa under Gal4-UAS control (UAS-cpa-IR). Flies expressing these constructs displayed similar phenotypes to flies mutant for the $c p a$ or $c p b$ allele (Janody and Treisman, 2006), including cell extrusion and 

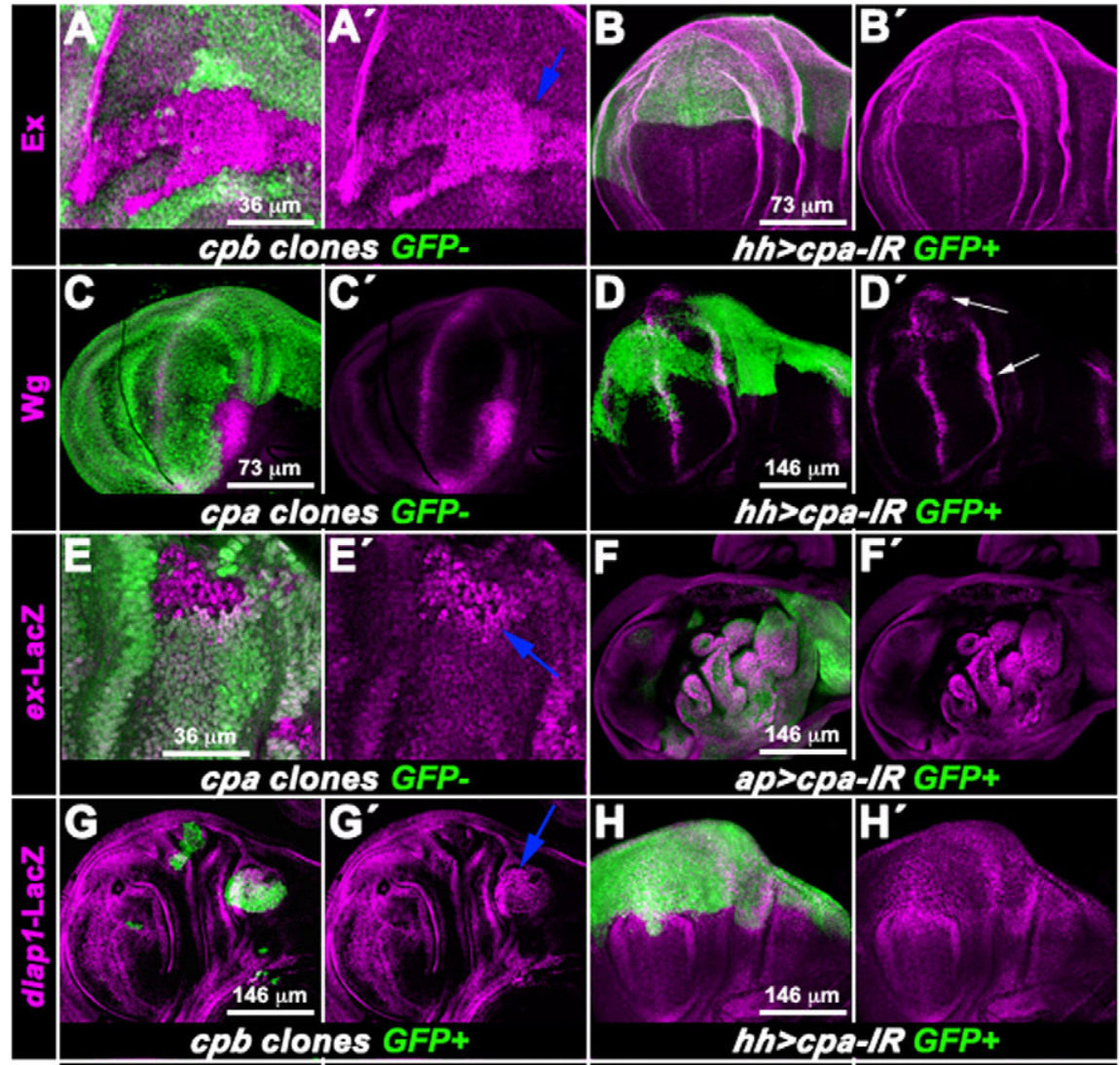

hh>cDa-lR GFP+
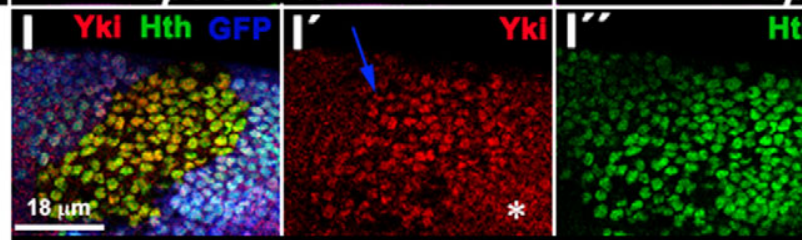

cpb clones GFP.

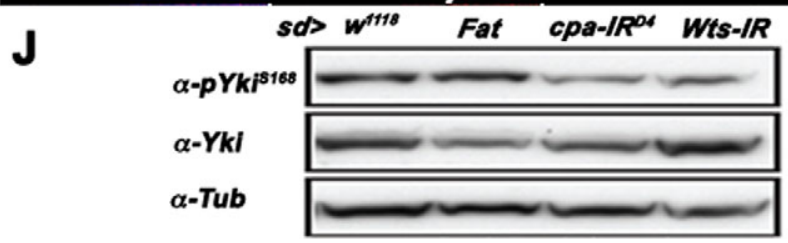

Fig. 2. Loss of CP leads to decreased phospho-Yki levels, promotion of Yki nuclear accumulation and upregulation of its target genes. (A-I'I') Standard confocal sections of Drosophila third instar wing imaginal discs showing $\mathrm{cpb} b^{\mathrm{M} 143}$

$\left(A, A^{\prime}, G, G^{\prime}, I-I^{\prime \prime \prime}\right), c^{107 E}\left(C, C^{\prime}\right)$ or cpa ${ }^{69 E}\left(E, E^{\prime}\right)$ mutant clones labeled negatively by the absence of GFP (green in A,C,E; blue in I, I') or positively labeled with GFP (green in G), and hh-Gal4 driving UAS-cpa-IRC10 and UAS-GFP $\left(\mathrm{B}, \mathrm{B}^{\prime}, \mathrm{D}, \mathrm{D}^{\prime}, \mathrm{H}, \mathrm{H}^{\prime}\right)$ or ap-Gal4 driving UAS-cpa$I R^{D 4}$ and UAS-GFP $\left(F, F^{\prime}\right)$. Discs are stained with anti-Ex $\left(A-B^{\prime}\right)$, anti-Wg $\left(C-D^{\prime}\right)$, anti- $\beta$ galactosidase $\left(\mathrm{E}-\mathrm{H}^{\prime}\right)$ to reveal ex-lacZ $\left(\mathrm{E}-\mathrm{F}^{\prime}\right)$ or $\operatorname{diap1-lacZ}\left(\mathrm{G}-\mathrm{H}^{\prime}\right)$, or anti-Yki (red in I, I') and anti-Hth (green in $I, I^{\prime \prime}$ ). Blue arrows (in $A^{\prime}$ ) indicate reduced Ex levels in wild-type cells adjacent to the cpb mutant clone or (in $\left.E^{\prime}, G^{\prime}, I^{\prime}\right)$ non-autonomous upregulation of exlacZ $\left(E^{\prime}\right)$, diap1-lacZ $\left(G^{\prime}\right)$ or Yki ( $\left.I^{\prime}\right)$ nuclear accumulation in wild-type cells adjacent to $C P$ mutant clones. The white asterisk in I' indicates the diffuse cytoplasmic and nuclear Yki localization in wild-type cells. (J) Protein extracts from wing discs carrying $s d-G a l 4$ $\left(w^{1118}\right.$, lane 1$)$, UAS-fat (lane 2), UAS-cpa-IR ${ }^{D 4}$ (lane 3) or UAS-wts-IR ${ }^{12072 R-1}$ (lane 4) and blotted with anti-pYki ${ }^{\text {S168A }}$ (upper panel), antiYki (middle panel) or anti- $\beta$-Tubulin (lower panel). Note that whereas Fat overexpression increases pYki level, reducing Cpa or Wts levels decreases pYki levels. death within the distal wing epithelium (see Fig. S1C-D in the supplementary material) and apical F-actin accumulation (see Fig. S1E-F in the supplementary material). To investigate the consequences of knocking down cpa on tissue growth in the proximal wing domain, we drove expression of $c p a-I R$ in the developing wing using wing disc-specific drivers. Expressing cpa$I R$ with apterous-Gal4 (ap-Gal4) induced abnormal growth of the dorsal wing disc compartment, characterized by the formation of extra folding (Fig. 1D; see Table S1 in the supplementary material). To quantify the amount of overgrowth, we expressed cpa-IR with a driver that would induce overgrowth of the proximal wing tissue without triggering tissue misfolding. scalloped-Gal4 (sd-Gal4) drives GFP expression in the whole wing disc primordium at early larval stages (Klein and Arias, 1998). By mid-third instar larvae, GFP was then restricted to the central BL and distal hinge (DH) domains (see Fig. S2A in the supplementary material) and progressively faded in this later region that co-expresses the two markers Nubbin (Nub) and Homothorax (Hth) (see Fig. S2B-C in the supplementary material). As expected, expressing dsRNA directed against Hpo with $s d$-Gal4 triggered an expansion of 55\% $(P<0.001)$ of the DH domain, as measured on cross-sections by an increase of the apical length of the Nub/Hth-positive domain (Fig. $1 \mathrm{H}$ and compare Fig. $1 \mathrm{G}$ with $1 \mathrm{E})$. Upon expression of two independent cpa dsRNA, the DH domain also showed a $21.5 \%$ and $26.2 \%$ increase $(P<0.03)$ in length, respectively (Fig. $1 \mathrm{H}$ and compare Fig. 1F with $1 \mathrm{E}$ ). We conclude that $\mathrm{CP}$ limits the growth of the distal hinge tissue.

To test the effects of loss of $\mathrm{CP}$ on cell proliferation, we analyzed the pattern of bromodeoxyuridine (BrdU) incorporation, which identifies cells undergoing DNA synthesis. We observed reproducible increases in BrdU labeling in cells expressing cpa-IR in the whole posterior compartment of the wing imaginal disc under hedgehog-Gal4 (hh-Gal4) control (Fig. 1I). We quantified this effect by measuring the intensity of BrdU signal in posterior versus anterior wing compartments in $h h>c p a-I R$ and control $h h>$ GFP wing discs. After normalization with $h h>$ GFP control 


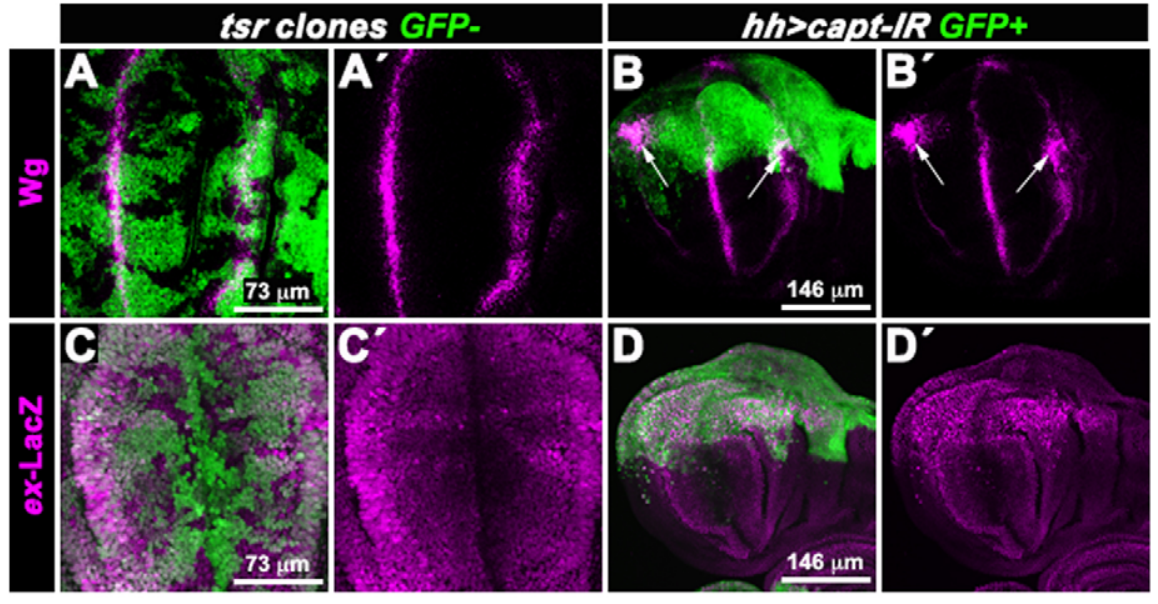

Fig. 3. Reducing levels of Capt but not Tsr increases transcription of Yki target genes. All panels show standard confocal sections of Drosophila third instar wing imaginal discs. $\left(\mathbf{A}, \mathbf{A}^{\prime}, \mathbf{C}, \mathbf{C}^{\prime}\right)$ Clones mutant for $\operatorname{tsr}^{110 M}\left(\mathrm{~A}, \mathrm{~A}^{\prime}\right)$ or $t_{s r}{ }^{76 E}\left(C, C^{\prime}\right)$ marked by the absence of GFP. $\left(\mathbf{B}^{\prime} \mathbf{B}^{\prime}, \mathbf{D}, \mathbf{D}^{\prime}\right)$ hh-Gal4 driving expression of UAScapt-IR $R^{5061 R-2}$ and UAS-GFP. Discs are stained with anti-Wg $\left(A-B^{\prime}\right)$ or anti- $\beta$-galactosidase to reveal ex-lacZ expression ( $\left.C-D^{\prime}\right)$. White arrows in $B, B^{\prime}$ indicate ectopic $W g$ expression. discs, we found that signal intensity was $34 \%$ higher $(P<0.004)$ in cells expressing cpa-IR (Fig. 1J). Taken together, these data indicate that CP limits cell proliferation in the whole wing disc epithelium but restricts tissue overgrowth only in the proximal wing disc epithelium.

\section{Capping Protein inhibits ectopic expression of Yorkie target genes}

The Hpo signaling pathway is a crucial regulator of tissue size (Edgar, 2006). We therefore tested whether loss of CP affects Hpo pathway target genes. Indeed, $c p a$ or $c p b$ mutant clones accumulated Ex (Fig. 2A) and Mer (see Fig. S4C in the supplementary material) at the apical cell surface (see Fig. S4B,D in the supplementary material) and $\mathrm{Wg}$ in the inner distal ring, within the proximal wing (Fig. 2C). Increase expression of Ex (Fig. 2B), Wg (Fig. 2D, arrows in $\mathrm{D}^{\prime}$ ) and Mer (see Fig. S2D in the supplementary material) could also be observed when we drove $c p a-I R$ with $h h$ or $a p$-Gal4. We then tested whether Ex upregulation occurs at the level of transcription. Expression of a lacZ enhancer trap insertion into the ex gene (ex-lacZ) was upregulated in $c p a$ mutant clones (Fig. 2E) and in $a p>c p a-I R$-expressing discs (Fig. $2 \mathrm{~F}$ ). Moreover, $c p b$ mutant clones (Fig. 2G) or $h h>c p a-I R-$ expressing cells (Fig. 2H) also upregulated expression of a lacZ enhancer trap insertion in the Diapl gene. Interestingly, we also noticed that loss of $\mathrm{CP}$ affected Hpo target genes non-cell autonomously. Whereas ex-lacZ and diapl-lacZ were upregulated in wild-type cells adjacent to the CP mutant tissue (blue arrows in Fig. 2E', $\mathrm{G}^{\prime}$ ), Ex protein levels were reduced (blue arrow in Fig. $2 \mathrm{~A})$. This suggests that $\mathrm{CP}$ affects $\mathrm{Yki}$ transcriptional activity cell autonomously but also non-autonomously in wild-type neighboring cells. In agreement, Yki colocalized with the nuclear transcription factor Hth in $c p b$ mutant clones (Fig. 2I) and in wild-type cells adjacent to the clone border (blue arrow in Fig. 2I'), whereas it showed a diffuse staining in wild-type cells located a few rows far from the mutant clone (white asterisk in Fig. 2I). We also analyzed by western blot phosphorylation of Yki at Ser168, a crucial phosphorylation site induced upon activation of the Hpo pathway (Dong et al., 2007). Like Wts-depleted tissues ( $s d>w t s-I R$ ), extracts from $s d>c p a-I R$ contained decreased phospho-Yki levels, but did not display changes in the total level of Yki (Fig. 2J). Thus, consistent with the observed changes in Yki localization, loss of $\mathrm{CP}$ results in a decrease in phosphorylated Yki and upregulation of Yki target genes. Moreover, we observed a strong enhancement of growth and $\mathrm{Wg}$ expression when we combined expression of cpa
dsRNA with hpo or wts dsRNA (see Fig. S3A-F and Table S1 in the supplementary material). By contrast, expressing a yki dsRNA or overexpressing hpo could suppress the growth of CP-depleted tissue (see Fig. S3G-K and Table S1 in the supplementary material). Together, we conclude that $\mathrm{CP}$ limits Yki nuclear import cell autonomously and non-cell autonomously, thereby inhibiting its transcriptional activity.

In the distal wing disc epithelium, loss of CP does not lead to tissue overgrowth but triggers cell extrusion and death (Janody and Treisman, 2006). We therefore wondered whether Yki target genes were also upregulated in dying CP mutant cells. To analyze the expression of Yki target genes in this region, we examined wing imaginal discs 36 hours after induction of $c p a$ or $c p b$ mutant clones, allowing us to recover mutant cells still present within the epithelium. In these cells, Ex (see Fig. S4A in the supplementary material) and Mer (see Fig. S4C in the supplementary material) were also upregulated. Moreover, in the eye imaginal disc, cpa or $c p b$ mutant cells also upregulated diap1-lacZ (see Fig. S4E in the supplementary material), Ex (see Fig. S4F,G in the supplementary material) and Mer (see Fig. S4H in the supplementary material). We therefore conclude that the effect of CP on Yki activity is not specific to the proximal wing disc epithelium. Although in the proximal wing disc epithelium, loss of $C P$ triggers upregulation of Yki target genes and tissue growth, in other tissues, such as in the distal wing epithelium, despite inhibition of Hpo signaling activity, other factors such as the polarity status might prevent $\mathrm{CP}$ mutant cells from surviving and growing.

\section{F-actin accumulation per se does not trigger activation of Yorkie target genes}

$\mathrm{CP}$ inhibits the turnover of actin monomers at the filament barbed end (Schafer et al., 1995). We therefore wondered whether accumulation of F-actin per se could disrupt Hpo signaling activity. We first examined mutations in the Cofilin homolog twinstar (tsr), which accumulates F-actin around the entire cell cortex (Janody and Treisman, 2006) (see Fig. S5A in the supplementary material). tsr mutant clones neither accumulated Wg (Fig. 3A), nor upregulated ex-lacZ (Fig. 3C). However, when we expressed a dsRNA directed against the cyclase-associated protein capulet (capt), which restricts actin filament accumulation near the apical surface (Janody and Treisman, 2006) (see Fig. S5B in the supplementary material), we saw that posterior $h h>$ capt-IRexpressing cells accumulated $\mathrm{Wg}$ (arrows in Fig. 3B) and upregulated ex-lacZ (Fig. 3D). These data indicate that Yki target 

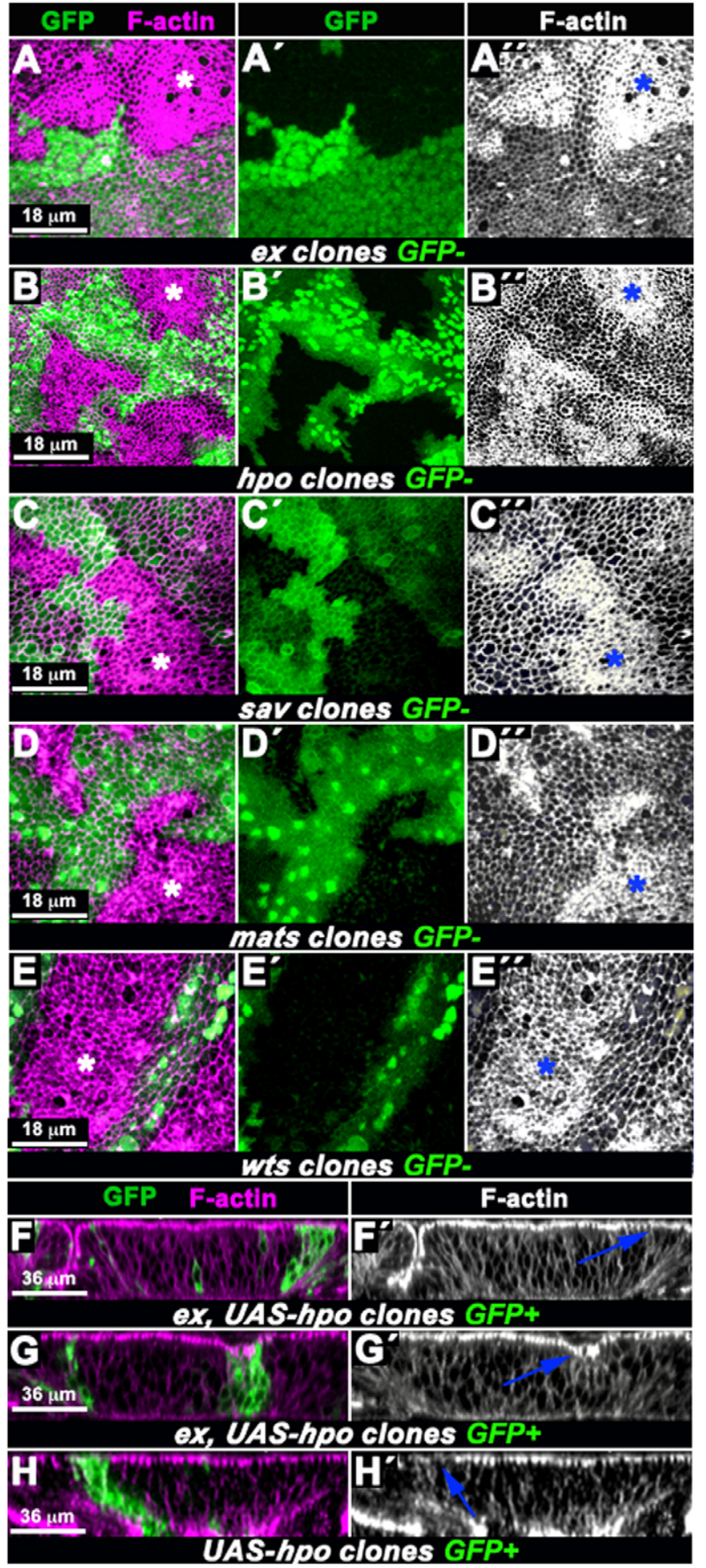

Fig. 4. Loss of ex, hpo, wts, mats or warts induces apical F-actin accumulation. All panels show Drosophila third instar wing imaginal discs stained with TRITC-Phalloidin to reveal F-actin. (A-E") Standard confocal sections of the apical surface of clones marked by the absence of GFP and mutant for ex ${ }^{e 1}\left(A-A^{\prime \prime}\right), h p o^{42-47}\left(B-B^{\prime \prime}\right)$, sav $\left(C-C^{\prime \prime}\right)$, mats ${ }^{2235}$ $\left(D-D^{\prime \prime}\right)$ or wts ${ }^{\text {lats } X 1}\left(E-E^{\prime \prime}\right)$. White and blue asterisks indicate F-actin accumulation in mutant cells. (F-H') Optical cross-sections through the disc epithelium of clones positively labeled with GFP, overexpressing UAS-hpo ${ }^{M 11.1}\left(\mathrm{H}, \mathrm{H}^{\prime}\right)$ and mutant for ex $\mathrm{e}^{\mathrm{e}}\left(\mathrm{F}-\mathrm{G}^{\prime}\right)$. Blue arrows indicate Factin levels in clones overexpressing $\mathrm{hpo}\left(\mathrm{H}^{\prime}\right)$ and mutant for $\mathrm{ex}^{\mathrm{e}}$ $\left(F^{\prime}, G^{\prime}\right)$. genes are transcriptionally upregulated when Capt levels are reduced. Together, these observations argue that accumulation of F-actin is not sufficient to trigger Yki activity, but support a role for an apical F-actin network in limiting Yki activity.

\section{Hippo signaling activity regulates F-actin independently of Yorkie}

Mammalian Mer has been shown to inhibit actin polymerization (McClatchey and Giovannini, 2005; Scoles, 2008). Moreover, the association of Merlin with the actin cytoskeleton is required to suppress growth (Curto and McClatchey, 2008). Because CP inhibits actin polymerization and limits Yki activity, one way by which Ex and Mer could regulate Hpo signaling activity is through the control of F-actin. We therefore wondered whether Drosophila Ex and Mer also have an effect on F-actin. When we induced ex mutant clones, we observed that, like cells in which Cpa levels were reduced (see Fig. S1E-F in the supplementary material), mutant cells accumulated F-actin near the apical surface (Fig. 4A; see Fig. S6A in the supplementary material). However, loss of Mer had only a weak effect on F-actin accumulation (see Fig. S7A-B in the supplementary material). Interestingly, clones mutant for hpo (Fig. 4B; see Fig. S6B in the supplementary material), sav (Fig. 4C; see Fig. S6C in the supplementary material), mats (Fig. 4D; see Fig. $\mathrm{S} 6 \mathrm{D}$ in the supplementary material) and $w t s$ (Fig. 4E; see Fig. S6E in the supplementary material) also accumulated apical F-actin, indicating that Ex and the whole kinase cassette inhibit F-actin formation. As Ex is an ERM family member, which can form a structural linkage between transmembrane components and the actin cytoskeleton (McClatchey and Giovannini, 2005), we then tested whether Ex prevents F-actin accumulation independently of its effect on the kinase Hpo. Overexpressing hpo suppressed growth and seemed to prevent $\mathrm{F}$-actin accumulation in ex mutant clones (blue arrow in Fig. 4F). Some clones constricted apically and appeared to contain increased apical F-actin (blue arrow in Fig. 4G). However, this effect is likely to result from a reduction of the apical cell surface in constricted tissues. Wild-type clones overexpressing hpo were also very small in size and seemed to slightly reduce apical F-actin (blue arrow in Fig. 4H). Therefore, the effect of Ex on F-actin might be through activation of the Hpo kinase. We conclude that Hpo pathway activity reduces F-actin at apical sites.

We then tested whether Hpo signaling activity controls F-actin through Yki activity. In wild-type clones, overexpressing Yki (Fig. $5 \mathrm{~A}$; see Fig. S6F in the supplementary material) or a constitutively active form of Yki $\left(y k i^{S 168 A}\right.$ ) (Fig. 5B; see Fig. S6G in the supplementary material) induced morphological defects but did not promote F-actin accumulation. Moreover, ex mutant clones in which Hpo signaling output is blocked by expressing yki dsRNA still accumulated F-actin at the apical surface (Fig. 5D; see Fig. S6I in the supplementary material), whereas wild-type clones expressing $y k i$ dsRNA did not significantly show disruption of the F-actin network (Fig. 5C; see Fig. S6H in the supplementary material). This indicates that Hpo signaling activity reduces apical F-actin independently of Yki.

\section{Hippo signaling activity and Capping Protein regulate apical F-actin}

An HA-tagged form of $\mathrm{Cpa}$, which can fully rescue cpa mutant animals to viability (Janody and Treisman, 2006), colocalized with Ex at apical sites (Fig. 6A). Moreover, both CP and Hpo signaling activities regulate apical $\mathrm{F}$-actin. We therefore asked whether increasing Hpo signaling modulates the effect of CP loss on Factin. Overexpression of a constitutively active form of $\mathrm{Mer}$ with 


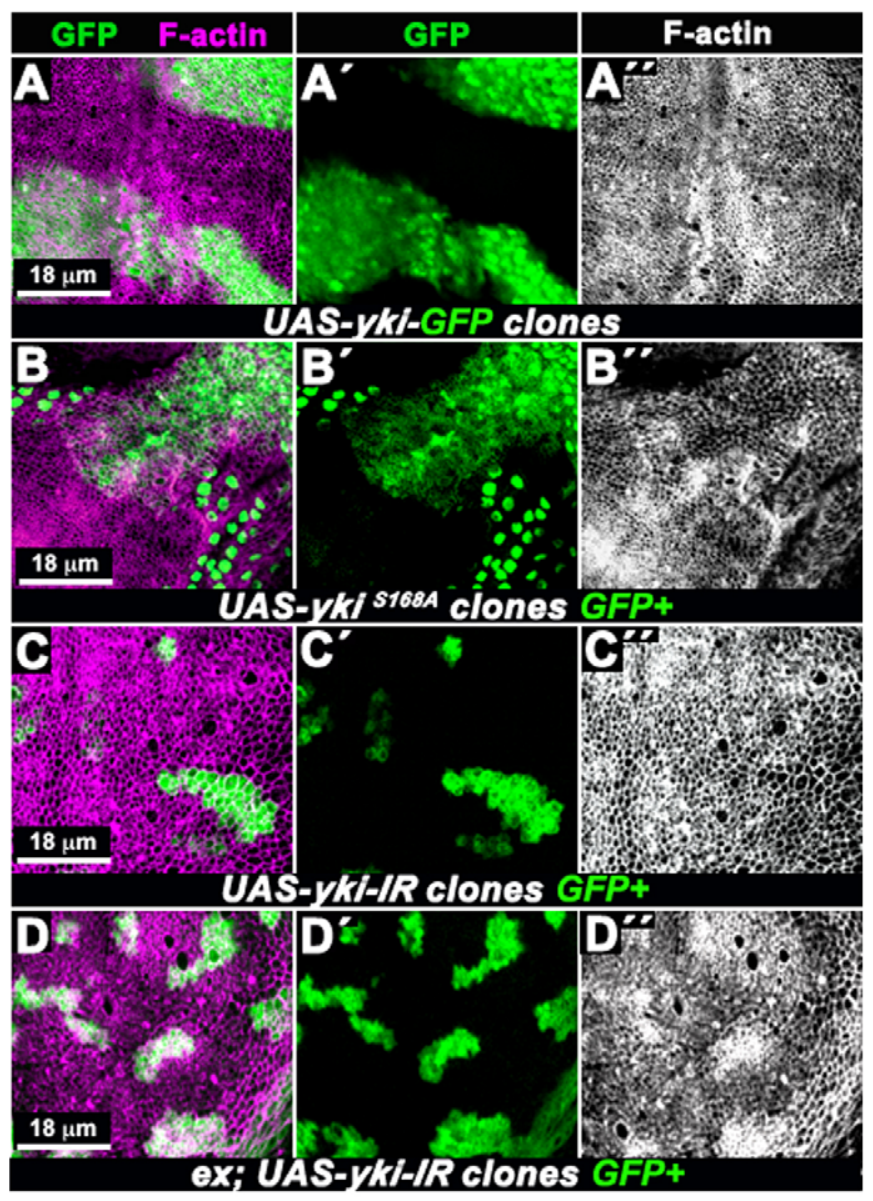

Fig. 5. Yki has no effect on F-actin levels. (A-D") All panels show standard confocal sections of the apical surface of Drosophila third instar wing imaginal discs stained with TRITC-Phalloidin (magenta in $A, B, C, D$ and white in $\left.A^{\prime \prime}, B^{\prime \prime}, C^{\prime \prime}, D^{\prime \prime}\right)$. Clones positively labeled with GFP (green in $\left.A, A^{\prime}, B, B^{\prime}, C, C^{\prime}, D, D^{\prime}\right)$ overexpressing UAS-yki-GFP $\left(A-A^{\prime \prime}\right)$, UAS$y k i^{S 168 A}\left(\mathrm{~B}-\mathrm{B}^{\prime \prime}\right)$ or UAS-yki-IR $R^{4005 R-2}\left(\mathrm{C}-\mathrm{C}^{\prime \prime}\right)$, or mutant for ex ${ }^{\mathrm{e}}\left(\mathrm{D}-\mathrm{D}^{\prime \prime}\right)$.

ap-Gal4 did not affect F-actin organization nor induced morphological defects (see Fig. S7C-D in the supplementary material). As cells overexpressing ex (Fig. 6B) or hpo (see Fig. $\mathrm{S} 3 \mathrm{H}$ in the supplementary material) grow very poorly, we induced the expression of these genes late in development using a thermosensitive form of Gal80 expressed under the control of the ubiquitous tubulin promoter ( $t u b-G a l 80^{\text {ts }}$ ) to inhibit GAL4 activity at earlier stages. $t u b-G a l 80^{\text {ts }}, h h$-Gal4; UAS-ex or UAS- $h p o$ larvae switched from $18^{\circ} \mathrm{C}$ to $25^{\circ} \mathrm{C}$ at early third instar and dissected 48 hours later maintained ex (Fig. 6C) or hpo (Fig. 6F)-overexpressing cells within the wing disc epithelium and did not show significant disruption of the apical F-actin network. By contrast, Gal $80^{\text {ts }}$; $h h>c p a-I R$ wing imaginal discs displayed increased F-actin in the posterior compartment (Fig. 6D). Interestingly, ex overexpression partially suppressed the F-actin accumulation caused by knocking down Cpa (Fig. 6E). We quantified this effect by measuring the ratio of Phalloidin signals between the posterior and anterior compartments for each genetic combination (Fig. $6 \mathrm{H}$ ). This ratio was 0.99 for Gal $80^{\text {ts. }}, h h>G F P$ control discs. By contrast, Gal $80^{\text {ts }}$; $h h>c p a-I R$ wing imaginal discs contained 1.82 times more F-actin at the apical surface of posterior cells. This actin accumulation was significantly reduced by ex overexpression (P/A ratio: 1.15,
$P<0.005)$, although ex overexpression in wild-type discs had no significant effect on F-actin. Overexpressing hpo also seemed to partially suppress F-actin accumulation of cpa-IR expressing cells $(P<0.07)$. These results indicate that Hpo signaling activity acts downstream or in parallel to $\mathrm{CP}$ on F-actin.

\section{DISCUSSION}

In this report, we show an interdependency between Hpo signaling activity and F-actin dynamics in which $\mathrm{CP}$ and Hpo pathway activities inhibit $\mathrm{F}$-actin accumulation, and the reduction in $\mathrm{F}$-actin in turn sustains Hpo pathway activity, preventing Yki nuclear translocation and upregulation of proliferation and survival genes (Fig. 7).

\section{Capping Protein and Hippo signaling activities inhibit F-actin}

ERM proteins can form a structural linkage between transmembrane components and the actin cytoskeleton (McClatchey and Giovannini, 2005). Mammalian Mer appears to act as an inhibitor of actin polymerization (McClatchey and Giovannini, 2005; Scoles, 2008). Moreover, the Mer-actin cytoskeleton association has a crucial role for growth suppression and inhibition of EGFR signaling (Curto and McClatchey, 2008). In Drosophila, Mer and Ex are structurally related and appear to have partially redundant functions but vary in their requirement depending of the tissue or developmental stage (Boedigheimer et al., 1993; Boedigheimer et al., 1997; Hamaratoglu et al., 2006; McCartney et al., 2000). In imaginal discs, loss of ex shows stronger phenotypes when compared with those of $\mathrm{Mer}$ (Boedigheimer et al., 1993; Boedigheimer et al., 1997; Hamaratoglu et al., 2006; McCartney et al., 2000). Ex might also have a stronger requirement on F-actin dynamics, as loss of ex, but not that of Mer, triggered F-actin accumulation (Fig. 4; see Figs S6, $\mathrm{S} 7$ in the supplementary material). Surprisingly, loss of hpo, sav, mats or wts also triggered apical F-actin accumulation (Fig. 4; see Fig. S6 in the supplementary material), independently of Yki activity (Fig. 5; see Fig. S6 in the supplementary material). Ex is likely to affect F-actin through activation of the Hpo kinase cassette because in most ex mutant clones, overexpressing hpo suppressed F-actin accumulation. Some clones seemed to contain increased Factin (Fig. 4). However, these clones also constricted apically, suggesting that the effect on $\mathrm{F}$-actin levels results from a reduction of the apical cell surface and that in the absence of $e x$, differential activity of overexpressed hpo triggers cell shape changes. Together, these observations argue that Ex prevents apical F-actin accumulation through Hpo signaling activity but independently of Yki.

Loss of Hpo pathway activity or CP triggered apical F-actin accumulation (Figs 4, 6; see Figs S1, S3, S6 in the supplementary material). Ex localizes to the sub-apical region of epithelial cells (Oh et al., 2009; Reddy and Irvine, 2008), and colocalized with an HA-tagged form of Cpa (Fig. 6), Ex, Hpo, Sav and Wts all interact with each other through WW and PPXY motifs (Oh et al., 2009; Reddy and Irvine, 2008). Therefore, a pool of Hpo, Sav and Wts, localized at apical sites, could directly regulate an actin-regulatory protein. Hpo pathway activity might act downstream of CP on Factin. In agreement with this, ex overexpression significantly suppressed F-actin accumulation in cells with reduced CP levels (Fig. 6). The role of Hpo signaling activity might be to inhibit an actin-nucleating factor, which adds new actin monomers to filament barbed ends free of the capping activity of CP. However, we cannot exclude that ex overexpression enhances the activity of 


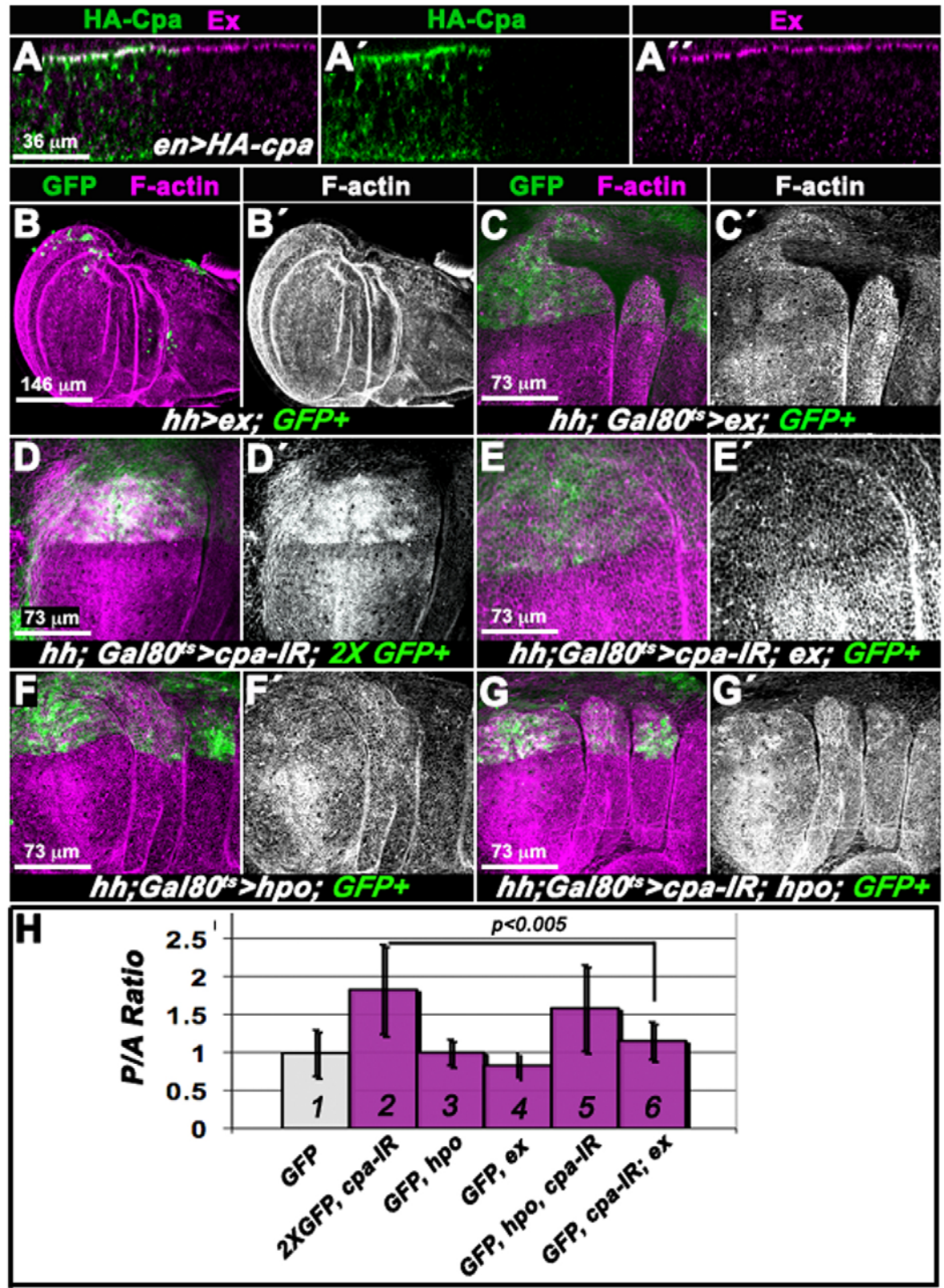

Fig. 6. Ex and CP colocalize at apical sites and regulate apical F-actin. All panels show Drosophila third instar wing imaginal discs. A-A" are optical cross-sections through the epithelium; B-G' are standard confocal sections. (A-A') en-Gal4 driving expression of UAS-HA-cpa. Discs are stained with anti-HA (green in $A, A^{\prime}$ ) and anti-Ex (magenta in $\left.A, A^{\prime \prime}\right)$. (B, $\left.\mathbf{B}^{\prime}\right)$ hh-Gal4 driving UAS-GFP (green in B) and UAS-ex. (C-G') tub-Gal80ts; hhGal4 driving one copy of UAS-GFP (green in $C)$ and UAS-ex $\left(C, C^{\prime}\right)$; two copies of UASGFP (green in D) and UAS-cpa-IR ${ }^{C 10}\left(\mathrm{D}, \mathrm{D}^{\prime}\right)$; one copy of UAS-GFP (green in E), UAS-cpa$I R^{C 10}$ and UAS-ex $\left(\mathrm{E}_{1} \mathrm{E}^{\prime}\right)$; one copy of UASGFP (green in F) and UAS-hpo M11.1 (F,F'); or one copy of UAS-GFP (green in G), UAS-cpa$I^{C 10}$ and UAS-hpo ${ }^{M 11.1}\left(\mathrm{G}, \mathrm{G}^{\prime}\right)$. Discs are stained with TRITC-Phalloidin (magenta in B$\mathrm{G}$ and white in $\left.\mathrm{B}^{\prime}-\mathrm{G}^{\prime}\right)$. (H) Mean intensity of the ratio of Phalloidin signal between posterior and anterior wing compartments (P/A ratio) of tub-Gal $80^{\text {ts. }}$, hh-Gal4 driving UAS-GFP (1; mean=0.99); UAS-cpa- $I R^{C 10}$ and two copies of UAS-GFP (2; mean=1.82); UAS-hpo ${ }^{\text {M11. } 1}$ and UAS-GFP (3; mean=1); UAS-ex and UAS-GFP (4; mean $=0.82)$; UAScpa-IR ${ }^{C 10}$, UAS-hpo ${ }^{M 11.1}$ and UAS-GFP (5; mean=1.58); or UAS-cpa-IRC10, UAS-ex and UAS-GFP (6; mean=1.15). Error bars indicate s.e.m. $P<0.005$ for comparison of $\mathrm{f} 2$ and 6 .

residual Cpa in cells knocked down using RNAi, nor that Hpo pathway activity acts in parallel to $\mathrm{CP}$ on F-actin. Interestingly, although endogenous Ex is upregulated in cells lacking CP (Fig. 2; see Fig. S4 in the supplementary material), mutant cells still accumulated F-actin (Janody and Treisman, 2006). wts mutant clones also upregulated Ex, which, when overexpressed, suppresses growth of wts mutant clones (Badouel et al., 2009). Therefore, the increased levels of endogenous Ex in cells lacking either CP or wts appears to be insufficient to fully suppress the effects of loss of $\mathrm{CP}$ or $w t s$ on F-actin and growth, respectively.

\section{Actin cytoskeleton genes control Hippo signaling activity}

Our data indicates that CP inhibits Yki nuclear accumulation, activation of Yki target genes, and consequently overgrowth of the proximal wing epithelium (Figs 1, 2; see Fig. S4 in the supplementary material). Interestingly, Yki was also found to accumulate in nuclei of wild-type cells adjacent to the clone border.
Consistent with a non-autonomous effect of $\mathrm{CP}$ loss on Hpo pathway activity, ex-lacZ and diap1-lac $Z$ were upregulated in wildtype cells adjacent to CP mutant clones. However, Ex levels were reduced in wild-type neighboring cells (Fig. 2). Cells expressing different amounts of $d s$ and $f j$ also upregulate ex-lacZ, but show reduced levels of Ex (Willecke et al., 2008). Therefore, loss of CP might affect Fj or Ds levels, creating a sharp boundary of their expression. However, in contrast to clones overexpressing $d s$ or mutant for $f j$, cell lacking CP also upregulated Ex and Mer inside the mutant clones, indicating that $\mathrm{CP}$ also acts cell-autonomously to promote $\mathrm{Hpo}$ signaling activity. $\mathrm{CP}$ might facilitate $\mathrm{Yki}$ phosphorylation by the Hpo kinase cassette as cpa-depleted tissues contained decreased phospho-Yki levels (Fig. 2). But, we cannot exclude the possibility that $\mathrm{CP}$ also favors the direct binding of non-phosphorylated Yki to Ex, Hpo or Wts (Oh et al., 2009). Further analysis will be required to elucidate the mechanisms by which CP restricts Yki activity cell autonomously and in wild-type neighboring cells. 


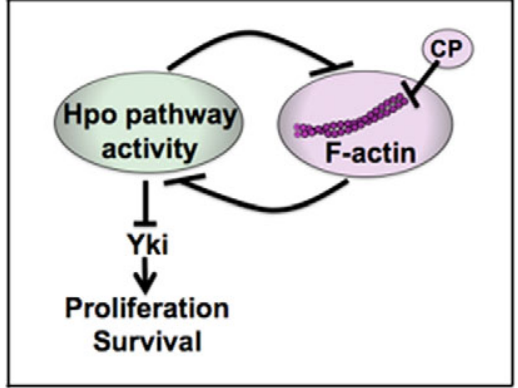

Fig. 7. Schematic of the interplay between Hpo pathway activity and F-actin dynamics. CP and Hpo pathway activities prevent F-actin accumulation, which would otherwise inhibit Hpo pathway activity, thereby promoting Yki nuclear translocation and upregulation of proliferation and survival genes.

Our results argue for a constitutive role of CP in Hpo pathway activity as Yki target genes were upregulated in the whole wing and eye imaginal discs (Fig. 2; see Fig. S4 in the supplementary material). However, loss of CP did not fully recapitulate the phenotype for core components of the hpo pathway (Edgar, 2006). Despite that, on average, $c p b$ mutant clones located in the proximal wing disc domain were $25 \%$ larger than wild-type twin spots; $60 \%$ of mutant clones failed to grow (Fig. 1; see Fig. S1 in the supplementary material). Moreover, in the distal wing epithelium, reducing $\mathrm{CP}$ levels induces mislocalization of the adherens junction components Armadillo and DE-Cadherin, extrude and death (Janody and Treisman, 2006) (see Fig. S1 in the supplementary material). Furthermore, in Drosophila, CP also prevents retinal degeneration (Delalle et al., 2005; Johnson et al., 2008). This indicates that although loss of CP can, under certain conditions, result in tissue overgrowth due to inhibition of Hpo pathway activity, other factors such as the polarity status, also determine the survival and growth of the mutant tissue. Therefore, in addition to promoting Hpo pathway activity, $\mathrm{CP}$ has additional developmental functions in epithelia. However, we cannot exclude the possibility that, like most upstream inputs that feed into the Hpo pathway (Halder and Johnson, 2010), CP has a tissue-specific requirement in Hpo pathway activity.

CP, Capt and Tsr all restrict F-actin assembly directly (Bamburg, 1999; Cooper and Sept, 2008; Gottwald et al., 1996). CP and Capt control F-actin formation near the apical surface and inhibit ectopic expression of Yki target genes, whereas Tsr acts around the entire cell cortex and has no effect on Yki target genes (Janody and Treisman, 2006) (Fig. 3; see Fig. S5 in the supplementary material). This argues that Hpo signaling activity is not affected by the excess of F-actin per se but provides significant support to the view that stabilization of an apical F-actin network by CP, Capt and Hpo signaling activity feeds back on the Hpo pathway to sustain its activity.

Our findings do not allow us to conclude where F-actin accumulation intersects Hpo signaling activity because both Hpo signaling activity and F-actin dynamics feedback to each other. For instance, hpo or ex overexpression suppressed growth of CPdepleted cells (see Fig. S3 in the supplementary material; data not shown). But, overexpressed ex and possibly hpo also suppressed Factin accumulation of Cpa-depleted cells (Fig. 6). The control of F-actin by Hpo signaling activity and CP might constitute a parallel input, which sustains Hpo pathway activity. Alternatively, F-actin could act upstream of the core kinase cascade, which in turn feeds back to F-actin, to maintain its activity. The identification of additional actin cytoskeletal components that either promote Hpo pathway activity or act downstream of Hpo pathway activity on Factin would help to discriminate between these possibilities.

\section{Mechanisms by which F-actin regulates Hippo signaling activity}

How F-actin influences Hpo signaling activity remains to be determined. The apical F-actin network, which regulates the formation and movement of endocytic vesicles from the plasma membrane (Smythe and Ayscough, 2006), might promote the recycling or degradation of Hpo pathway components. Increased F-actin at apical sites would, therefore, affect protein turnover. Alternatively, apical F-actin might act as a scaffold to tether Hpo pathway components apically. In support of this, Ex, Hpo, Sav, Wts and Yki could all interact between each other through WW and PPXY motifs at apical sites (Oh et al., 2009; Reddy and Irvine, 2008). Moreover, expression of a membrane-targeted form of Mats enhances Hpo signaling (Ho et al., 2010). Although Ex and Mer were properly localized in CP mutant cells (see Fig. S4 in the supplementary material), other members of the pathway might be mislocalized in the presence of excess Factin. Interestingly, in mouse fibroblasts, the Hpo orthologs MST1 and MST2 colocalize with F-actin structures and are activated upon F-actin depolymerization (Densham et al., 2009), suggesting that by tethering Hpo pathway components, F-actin dynamics modulates their activity. Finally, the F-actin network might act as a mechanical transducer. Most of the mechanosensitive responses require tethering to force-bearing actin filaments (Vogel and Sheetz, 2006). Tissue surface tension has been proposed to be a stimulus for a feedback mechanism that could regulate tissue growth (Aegerter-Wilmsen et al., 2007; Hufnagel et al., 2007). The tension exerted by neighboring cells might be sensed at the cell membrane by the actin cytoskeleton and translated to the regulation of cell proliferation through the Hpo signaling pathway.

\section{Acknowledgements}

We thank S. Cohen, R. Mann, A. Laughon, A. Salzberg, B. Hay, N. Tapon, K. D. Irvine, D. J. Pan, R. G. Fehon, the Bloomington Drosophila Stock Center, the Drosophila Genomics Resource Center, the Vienna Drosophila Research Center (VDRC), the National Institute of Genetics (NIG) and the Developmental Studies Hybridoma Bank (DSHB) for fly stocks and reagents. The manuscript was improved by the critical comments of Nicolas Tapon, Jessica Treisman, Francesca Pignoni, Monica Bettencourt-Dias and Moises Mallo. This work was supported by grants (PTDC/SAU-OBD/73191/2006 and PTDC/BIA-BCM/ 71674/2006) from Fundação para a Ciência e Tecnologia (FCT). B.G.F., P.G., C.B.-P. and B.J. were the recipient of fellowships from FCT (SFRH/BPD/ 35915/2007, SFRH/BD/47261/2008, SFRH/BPD/46983/2008 and SFRH/BD/ 33215/2007, respectively).

\section{Competing interests statement}

The authors declare no competing financial interests.

\section{Supplementary material}

Supplementary material for this article is available at

http://dev.biologists.org/lookup/suppl/doi:10.1242/dev.063545/-/DC1

\section{References}

Aegerter-Wilmsen, T., Aegerter, C. M., Hafen, E. and Basler, K. (2007). Model for the regulation of size in the wing imaginal disc of Drosophila. Mech. Dev. 124, 318-326

Badouel, C., Gardano, L., Amin, N., Garg, A., Rosenfeld, R., Le Bihan, T. and McNeill, H. (2009). The FERM-domain protein Expanded regulates Hippo pathway activity via direct interactions with the transcriptional activator Yorkie. Dev. Cell 16, 411-420

Bamburg, J. R. (1999). Proteins of the ADF/cofilin family: essential regulators of actin dynamics. Annu. Rev. Cell Dev. Biol. 15, 185-230. 
Bennett, F. C. and Harvey, K. F. (2006). Fat cadherin modulates organ size in Drosophila via the Salvador/Warts/Hippo signaling pathway. Curr. Biol. 16, 21012110

Boedigheimer, M., Bryant, P. and Laughon, A. (1993). Expanded, a negative regulator of cell proliferation in Drosophila, shows homology to the NF2 tumor suppressor. Mech. Dev. 44, 83-84

Boedigheimer, M. J., Nguyen, K. P. and Bryant, P. J. (1997). Expanded functions in the apical cell domain to regulate the growth rate of imaginal discs. Dev. Genet. 20, 103-110.

Bretscher, A., Edwards, K. and Fehon, R. G. (2002). ERM proteins and merlin: integrators at the cell cortex. Nat. Rev. Mol. Cell Biol. 3, 586-599.

Cho, E., Feng, Y., Rauskolb, C., Maitra, S., Fehon, R. and Irvine, K. D. (2006). Delineation of a Fat tumor suppressor pathway. Nat. Genet. 38, 11421150 .

Cooper, J. A. and Sept, D. (2008). New insights into mechanism and regulation of actin capping protein. Int. Rev. Cell Mol. Biol. 267, 183-206.

Curto, M. and McClatchey, A. I. (2008). Nf2/Merlin: a coordinator of receptor signalling and intercellular contact. Br. J. Cancer 98, 256-262.

Delalle, I., Pfleger, C. M., Buff, E., Lueras, P. and Hariharan, I. K. (2005) Mutations in the Drosophila orthologs of the F-actin capping protein alpha- and beta-subunits cause actin accumulation and subsequent retinal degeneration. Genetics 171, 1757-1765.

Densham, R. M., O’Neill, E., Munro, J., Konig, I., Anderson, K., Kolch, W. and Olson, M. F. (2009). MST kinases monitor actin cytoskeletal integrity and signal via c-Jun $\mathrm{N}$-terminal kinase stress-activated kinase to regulate p21Waf1/Cip1 stability. Mol. Cell. Biol. 29, 6380-6390.

Dong, J., Feldmann, G., Huang, J., Wu, S., Zhang, N., Comerford, S. A., Gayyed, M. F., Anders, R. A., Maitra, A. and Pan, D. (2007). Elucidation of universal size-control mechanism in Drosophila and mammals. Cell 130, 11201133.

Edgar, B. A. (2006). From cell structure to transcription: Hippo forges a new path Cell 124, 267-273.

Gottwald, U., Brokamp, R., Karakesisoglou, I., Schleicher, M. and Noegel, A. A. (1996). Identification of a cyclase-associated protein (CAP) homologue in Dictyostelium discoideum and characterization of its interaction with actin. $\mathrm{Mol}$. Biol. Cell 7, 261-272

Grusche, F. A., Richardson, H. E. and Harvey, K. F. (2010). Upstream regulation of the hippo size control pathway. Curr. Biol. 20, R574-R582.

Halder, G. and Johnson, R. L. (2010). Hippo signaling: growth control and beyond. Development 138, 9-22.

Hamaratoglu, F., Willecke, M., Kango-Singh, M., Nolo, R., Hyun, E., Tao, C., Jafar-Nejad, H. and Halder, G. (2006). The tumour-suppressor genes NF2/Merlin and Expanded act through Hippo signalling to regulate cell proliferation and apoptosis. Nat. Cell Biol. 8, 27-36.

Hay, B. A., Wassarman, D. A. and Rubin, G. M. (1995). Drosophila homologs of baculovirus inhibitor of apoptosis proteins function to block cell death. Cell $\mathbf{8 3}$ 1253-1262.

Ho, L. L., Wei, X., Shimizu, T. and Lai, Z. C. (2010). Mob as tumor suppressor is activated at the cell membrane to control tissue growth and organ size in Drosophila. Dev. Biol. 337, 274-283

Huang, J., Wu, S., Barrera, J., Matthews, K. and Pan, D. (2005). The Hippo signaling pathway coordinately regulates cell proliferation and apoptosis by inactivating Yorkie, the Drosophila Homolog of YAP. Cell 122, 421-434

Hufnagel, L., Teleman, A. A., Rouault, H., Cohen, S. M. and Shraiman, B. I. (2007). On the mechanism of wing size determination in fly development. Proc. Natl. Acad. Sci. USA 104, 3835-3840

Janody, F. and Treisman, J. E. (2006). Actin capping protein \{alpha\} maintains vestigial-expressing cells within the Drosophila wing disc epithelium. Development 133, 3349-3357.

Johnson, R. I., Seppa, M. J. and Cagan, R. L. (2008). The Drosophila CD2AP/CIN85 orthologue Cindr regulates junctions and cytoskeleton dynamics during tissue patterning. J. Cell Biol. 180, 1191-1204.

Johnston, L. A. and Schubiger, G. (1996). Ectopic expression of wingless in imaginal discs interferes with decapentaplegic expression and alters cell determination. Development 122, 3519-3529.

Klein, T. and Arias, A. M. (1998). Different spatial and temporal interactions between Notch, wingless, and vestigial specify proximal and distal pattern elements of the wing in Drosophila. Dev. Biol. 194, 196-212.
Lai, Z. C., Wei, X., Shimizu, T., Ramos, E., Rohrbaugh, M., Nikolaidis, N., Ho, L. L. and Li, Y. (2005). Control of cell proliferation and apoptosis by mob as tumor suppressor, mats. Cell 120, 675-685

LaJeunesse, D. R., McCartney, B. M. and Fehon, R. G. (1998). Structural analysis of Drosophila merlin reveals functional domains important for growth control and subcellular localization. J. Cell Biol. 141, 1589-1599.

Lee, J. D. and Treisman, J. E. (2001). Sightless has homology to transmembrane acyltransferases and is required to generate active Hedgehog protein. Curr. Biol. 11, 1147-1152

Lee, Y. S. and Carthew, R. W. (2003). Making a better RNAi vector for Drosophila: use of intron spacers. Methods 30, 322-329.

McCartney, B. M., Kulikauskas, R. M., LaJeunesse, D. R. and Fehon, R. G. (2000). The neurofibromatosis-2 homologue, Merlin, and the tumor suppressor expanded function together in Drosophila to regulate cell proliferation and differentiation. Development 127, 1315-1324.

McClatchey, A. I. and Giovannini, M. (2005). Membrane organization and tumorigenesis-the NF2 tumor suppressor, Merlin. Genes Dev. 19, 2265-2277.

Naito, Y., Yamada, T., Matsumiya, T., Ui-Tei, K., Saigo, K. and Morishita, S. (2005). dsCheck: highly sensitive off-target search software for double-stranded RNA-mediated RNA interference. Nucleic Acids Res. 33, W589-W591.

Oh, H. and Irvine, K. D. (2008). In vivo regulation of Yorkie phosphorylation and localization. Development 135, 1081-1088.

Oh, H., Reddy, B. V. and Irvine, K. D. (2009). Phosphorylation-independent repression of Yorkie in Fat-Hippo signaling. Dev. Biol. 335, 188-197.

Pantalacci, S., Tapon, N. and Leopold, P. (2003). The Salvador partner Hippo promotes apoptosis and cell-cycle exit in Drosophila. Nat. Cell Biol. 5, 921-927.

Reddy, B. V. and Irvine, K. D. (2008). The Fat and Warts signaling pathways: new insights into their regulation, mechanism and conservation. Development 135 , 2827-2838.

Schafer, D. A., Hug, C. and Cooper, J. A. (1995). Inhibition of CapZ during myofibrillogenesis alters assembly of actin filaments. J. Cell Biol. 128, 61-70.

Scoles, D. R. (2008). The merlin interacting proteins reveal multiple targets for NF2 therapy. Biochim. Biophys. Acta 1785, 32-54.

Silva, E., Tsatskis, Y., Gardano, L., Tapon, N. and McNeill, H. (2006). The tumor-suppressor gene fat controls tissue growth upstream of expanded in the hippo signaling pathway. Curr. Biol. 16, 2081-2089.

Smythe, E. and Ayscough, K. R. (2006). Actin regulation in endocytosis. J. Cell Sci. 119, 4589-4598.

Sopko, R., Silva, E., Clayton, L., Gardano, L., Barrios-Rodiles, M., Wrana, J., Varelas, X., Arbouzova, N. I., Shaw, S., Saburi, S. et al. (2009).

Phosphorylation of the tumor suppressor fat is regulated by its ligand Dachsous and the kinase discs overgrown. Curr. Biol. 19, 1112-1117

Spradling, A. C. and Rubin, G. M. (1982). Transposition of cloned P elements into Drosophila germ line chromosomes. Science 218, 341-347.

Tapon, N., Harvey, K. F., Bell, D. W., Wahrer, D. C., Schiripo, T. A., Haber, D. A. and Hariharan, I. K. (2002). salvador Promotes both cell cycle exit and apoptosis in Drosophila and is mutated in human cancer cell lines. Cell 110, 467-478.

Udan, R. S., Kango-Singh, M., Nolo, R., Tao, C. and Halder, G. (2003). Hippo promotes proliferation arrest and apoptosis in the Salvador/Warts pathway. Nat Cell Biol. 5, 914-920.

Vogel, V. and Sheetz, M. (2006). Local force and geometry sensing regulate cell functions. Nat. Rev. Mol. Cell Biol. 7, 265-275.

Wei, X., Shimizu, T. and Lai, Z. C. (2007). Mob as tumor suppressor is activated by Hippo kinase for growth inhibition in Drosophila. EMBO J. 26, 1772-1781.

Willecke, M., Hamaratoglu, F., Kango-Singh, M., Udan, R., Chen, C. L., Tao, C., Zhang, X. and Halder, G. (2006). The fat cadherin acts through the hippo tumor-suppressor pathway to regulate tissue size. Curr. Biol. 16, 2090-2100.

Willecke, M., Hamaratoglu, F., Sansores-Garcia, L., Tao, C. and Halder, G. (2008). Boundaries of Dachsous Cadherin activity modulate the Hippo signaling pathway to induce cell proliferation. Proc. Natl. Acad. Sci. USA 105, 1489714902.

Wu, S., Huang, J., Dong, J. and Pan, D. (2003). hippo encodes a Ste-20 family protein kinase that restricts cell proliferation and promotes apoptosis in conjunction with salvador and warts. Cell 114, 445-456.

Xu, T., Wang, W., Zhang, S., Stewart, R. A. and Yu, W. (1995). Identifying tumor suppressors in genetic mosaics: the Drosophila lats gene encodes a putative protein kinase. Development 121, 1053-1063. 